\title{
Soldadura cerámica: método de reparación de revestimientos refractarios de equipamientos industriales
}

\author{
H. E. LOBATO, M. A. CAMERUCCI
}

Laboratorio Materiales Estructurales-División Cerámicos-INTEMA-Fac. Ingeniería-UNMdP-CONICET. J. B. Justo 4302-(B7608FDQ) Mar del Plata-Argentina.

\begin{abstract}
El proceso de soldadura cerámica es un atractivo método para la reparación en caliente de revestimientos refractarios de equipamientos industriales. Consiste en la proyección a alta temperatura de una mezcla de soldadura en polvo mediante una corriente rica en oxígeno sobre el material refractario dañado. La mezcla contiene agregados refractarios gruesos compatibles con el sustrato a reparar y un combustible sólido (finas partículas oxidables, metálicas y/o no metálicas) que actúa como agente exotérmico en el proceso. Cuando las partículas combustibles impactan en la superficie caliente del sustrato $\left(1000-1500^{\circ} \mathrm{C}\right)$, reaccionan con el oxígeno liberando calor y alcanzando localmente temperaturas de $2000-2500^{\circ} \mathrm{C}$. Estas temperaturas producen la fusión parcial tanto del sustrato como de la carga de soldadura formándose una unión cerámica entre ambos.

Existe muy escasa bibliografía sobre el tema y la mayor parte de la información está protegida por patentes, existiendo ciertas discrepancias entre las disponibles. En este trabajo se presentan los principales aspectos de las investigaciones sobre el tema que han sido relevadas, incluyendo resultados y conclusiones de un estudio desarrollado sobre soldadura cerámica a escala laboratorio para la reparación de ladrillos de sílice de uso siderúrgico.
\end{abstract}

Palabras clave: refractarios; métodos de reparación; soldadura cerámica; hornos de coquización.

Ceramic welding: reparation method of refractory linings of industrial equipment.

The ceramic welding process is a unique hot repair technique for refractory linings in industrial equipments. This process consists of projecting a weld mix in powder by means of a current of oxygen or oxygen/air onto the refractory surface to be restored. The weld mix content refractory particles chemically compatible with the refractory to be repaired and a solid combustible (oxidizable fine particles, metallic and/or non metallic) as exotermic agent. When the combustible particles ignites at contact with the hot face $\left(1000-1500^{\circ} \mathrm{C}\right)$, an exotermic reaction with oxygen occurs developing temperatures of the order of $2000-2500^{\circ} \mathrm{C}$. The heat generated from this reaction is enough to melt the surface to be repaired and allowing a very close bond to be formed between the repaired area and the deposited material.

There are limited data in literature about ceramic welding and the larger part of the information is protected by patents. Several differences between the available patents were detected. In this work, the principal aspects of researches about ceramic welding that had been relieved are present, including results and conclusions from a study accomplished about ceramic welding (design and set up of a test in laboratory, and evaluation of the reparations) for the reparation of silica bricks employed to build coke ovens.

Keywords: refractories; reparation method; ceramic welding; coke ovens.

\section{INTRODUCCIÓN}

Los revestimientos refractarios de hornos y otros equipamientos son ampliamente utilizados en muy diversas industrias tales como las del acero, metales no ferrosos, vidrio y cemento. Habitualmente, son sometidos en servicio a severas condiciones (altas temperaturas, atmósferas oxidantes o reductoras, contacto con fundidos, entre otras), las cuales son causales de un inevitable y progresivo deterioro de origen térmico, mecánico y/o químico. Así, resulta de fundamental importancia, tanto por los altos costos involucrados como desde el punto de vista medioambiental, contar con métodos de reparación que permitan extender la vida útil de un revestimiento refractario $\sin$ la necesidad de realizar recambios parciales de los mismos.

Dentro de los métodos tradicionalmente empleados para la reparación de revestimientos refractarios se encuentran la pulverización húmeda ('wet spraying'), el emparchado ('patching') y la proyección con pistola a presión ('gunning'). Estos métodos consisten en proyectar con aire comprimido sobre el refractario dañado una mezcla de agua y un material refractario monolítico que contiene agregados refractarios, cementos y plastificantes (fosfatos, silicatos, arcillas). En todos ellos, independientemente de la temperatura del sustrato, se produce un anclaje mecánico entre éste y el material monolítico formándose unión cerámica recién durante la permanencia 
de la reparación a la temperatura de operación del horno. Si bien estos métodos involucran operaciones relativamente sencillas, de bajo costo y rápida aplicación, presentan serios inconvenientes: generación de tensiones de origen térmico en la interfase debido al desajuste entre los coeficientes de expansión térmica de las reparaciones y de los refractarios y fisuración originada por la alta proporción de agua empleada (10-60\%, dependiendo del método). En consecuencia, la vida útil de las reparaciones es limitada, ocasionando así altos costos. Por otro lado, el recambio parcial de ladrillos, si bien es un método efectivo, requiere de tiempos prolongados durante los cuales el horno permanecerá inactivo, y ciclos térmicos controlados en las paradas y puesta en marcha.

En este contexto, el proceso de soldadura cerámica se presenta como un atractivo método de reparación en caliente de revestimientos refractarios de equipamientos industriales. Este método fue desarrollado y originalmente diseñado en los años '70, por el grupo vidriero belga Glaverbel S.A. para hornos de la industria del vidrio. En 1977 esta técnica se evaluó en hornos de coquización en la industria del acero inglesa y en 1979 se introdujo en Estados Unidos como método de reparación de paredes de hornos de coque siderúrgico. Sin embargo, por sus elevados costos y la falta de experiencia en las técnicas de aplicación, su uso se extendió recién al final de la década de los '80 a Canadá, Europa, Japón y América Latina. Desde entonces, el proceso ha evolucionado para su aplicación en otras industrias tales como las del aluminio, cobre, cemento y fundiciones $(2,3)$.

Esta técnica presenta muchas ventajas, entre ellas: permite reparar defectos de muy diversos tipos (sellado de fisuras, grietas y juntas de expansión o ladrillos separados, paredes desalineadas, llenado de agujeros o de superficies deterioradas por pérdida de fragmentos por choque térmico o por desgaste por abrasión o erosión, sellado de escalones entre estructuras de ladrillos viejos y nuevos), tamaños y localización dentro del horno; puede ser integrada fácilmente a una rutina de mantenimiento y se realiza a las altas temperaturas de operación de los hornos, reduciéndose al mínimo los costos operacionales debido a las paradas de planta. Si bien los costos involucrados en el proceso son mayores que los correspondientes a los métodos convencionales de reparación de refractarios, se ven compensados por originar reparaciones con alta resistencia mecánica inmediatamente después de aplicada la mezcla y prolongada duración (no inferior a tres años) (1).

\section{SOLDADURA CERÁMICA}

\subsection{Descripción del método}

El proceso de soldadura cerámica consiste en proyectar sobre un material refractario dañado, a alta temperatura, una mezcla de soldadura en polvo constituida por agregados refractarios de composición similar a la del sustrato a reparar y partículas combustibles (metálicas y / o no metálicas) finamente divididas, las cuales actúan como agentes exotérmicos en el proceso. La proyección del polvo se realiza mediante corriente de oxígeno o de una mezcla oxígeno/aire, de acuerdo a la calidad de la reparación que se desee obtener. A la temperatura del horno en una atmósfera rica en oxígeno, las partículas combustibles proyectadas sobre la superficie del sustrato refractario dañado se oxidan liberando calor (reacciones altamente exotérmicas) alcanzándose localmente temperaturas de $2000-2500^{\circ} \mathrm{C}(1-11)$. Estas elevadas temperaturas producen la fusión parcial de la superficie del sustrato refractario y de las partículas refractarias de la mezcla de soldadura posibilitando la formación de uniones cerámicas entre ambos. La formación de una unión cerámica y la similitud de las propiedades del sustrato refractario y la masa de reparación formada hacen altamente eficaz el proceso aumentando la duración de las reparaciones hasta años antes de fallar. Para asegurar la formación de uniones de alta resistencia se debe tener en cuenta el grado de preparación de la superficie del sustrato a reparar y sus características (5). El espesor deseado de la masa de soldadura, se logra aplicando el material de reparación sucesivamente en capas sobre el área dañada. Para optimizar el proceso, el combustible debe ser confinado al área de reparación y las corrientes convectivas que puedan existir en el horno deben ser minimizadas ya que pueden desviar el flujo de material. El porcentaje de oxígeno utilizado debe ser al menos igual al requerimiento estequiométrico para la reacción de oxidación del combustible de la mezcla.

Las principales variables del proceso son el tipo, las características granulométricas (distribución de tamaños y tamaño medio de partícula) y el contenido del material refractario y del combustible en la mezcla de soldadura; la relación de caudales $\left(Q_{\text {gas transporte }} / Q_{\text {partículas provectadas }}\right)$; la temperatura del refractario a reparar (desde temperatura ambiente hasta $1700^{\circ} \mathrm{C}(4)$, dependiendo del tipo y características del sustrato refractario) y la velocidad y distancia de proyección.

\subsection{Dispositivos de aplicación}

Las máquinas que se han desarrollado para realizar reparaciones por soldadura cerámica son simples y seguras de operar. Consisten en un contenedor de la mezcla en polvo, un sistema de arrastre del material, equipos de control y una lanza refrigerada con agua para proyectar el material. Se pueden categorizar en dos tipos: máquinas presurizadas y máquinas abiertas tipo venturi.

La máquina presurizada utiliza una tolva a presión con oxígeno puro que transporta la mezcla a la zona a reparar. Este tipo de sistema no tiene ninguna abertura directa a la atmósfera, de modo que no arrastra aire en la corriente de material. Además, al ser un sistema presurizado, no es sensible a las diferencias de presión que se generan al elevar la lanza de reparación a determinada altura, pudiendo reparar así defectos en cualquier ubicación dentro del horno.

El sistema abierto utiliza una corriente de oxígeno para transportar la mezcla de soldadura a través de un sistema venturi. Este sistema arrastra aire atmosférico junto con la mezcla de soldadura como parte del efecto venturi. El aire que ingresa con el material provoca un aumento de $50-75 \%$ en la velocidad de las partículas lo cual interfiere con la completa combustión de las partículas, afectando a la reparación en dos aspectos: aumento del rebote de la mezcla y deterioro de las propiedades físicas de la masa de reparación. Adicionalmente, el nitrógeno del aire disminuye la efectividad de la combustión de las partículas ya que absorbe parte de la energía térmica que normalmente es empleada en el calentamiento de las partículas cerámicas. Debido a que los sistemas venturi son sensibles a las diferencias de presión, la longitud a la cual se eleva la lanza de reparación es un factor limitante en el uso de este sistema.

Con equipos industriales las velocidades de flujo de material que se alcanzan con un sistema cerrado son de 0,2 a $0,9 \mathrm{~kg} / \mathrm{min}$, mientras que con un sistema abierto se pueden 
alcanzar velocidades de 0,7-11,3 $\mathrm{kg} / \mathrm{min}$, dependiendo del diseño del equipamiento (6). A modo de ejemplo, en una patente inglesa $\left(\mathrm{BP} \mathrm{N}^{\circ} 2173715\right)$ se especifica que la velocidad de flujo del polvo fue de $1 \mathrm{~kg} / \mathrm{min}$ empleando un flujo de oxígeno de $550 \mathrm{nl} / \mathrm{min}$.

En la elección del tipo de máquina a emplear para realizar una reparación por soldadura cerámica se deben tener en cuenta diversos factores: tiempo de aplicación, calidad de la reparación y riesgo de una posible contaminación. El sistema abierto, a pesar de las desventajas señaladas, resulta muy adecuado cuando el factor limitante es el tiempo y el riesgo de contaminaciones a causa del rebote de material es bajo. Este tipo de máquinas resultan muy adecuadas para reparar refractarios en hornos de coque y tanques regeneradores de vidrio. Los sistemas cerrados son en general empleados cuando se requieren reparaciones de alta calidad, por ejemplo, en cámaras de fusión de vidrio (6).

\subsection{Materiales: sustratos refractarios para reparación y mezclas de soldadura cerámica}

Todos los materiales refractarios se pueden reparar por soldadura cerámica. Sin embargo, cada tipo de refractario requiere mezclas de soldadura cerámica y métodos de aplicación particulares (5). Para seleccionar la carga refractaria y el combustible sólido que forman la mezcla de soldadura cerámica se debe conocer cuál es el material refractario a reparar y considerar que una vez aplicados se genere una masa de reparación con composición química y propiedades similares a las del refractario base. Así, se disminuye el daño de origen térmico del sistema sustrato-interfase-soldadura y se asegura la adhesión de la masa formada al sustrato refractario.

Además de la composición de las mezclas de soldadura, sus características granulométricas inciden fuertemente en la eficacia del proceso y en la calidad de las reparaciones obtenidas. Respecto a este punto, existen ciertas discrepancias en la información disponible. En una patente inglesa (BP $\mathrm{N}^{\circ}$ 1330894), se encuentra que el tamaño medio de las partículas refractarias $(500 \mu \mathrm{m})$ debe ser mayor que el correspondiente al material oxidable $(50 \mu \mathrm{m})$ y el ancho de la distribución debe superar un valor mínimo. Una distribución amplia de tamaños contribuye a la formación de reparaciones duraderas, menos porosas y con menor cantidad de fisuras que las producidas usando partículas refractarias de tamaño más uniforme (distribución angosta). En otra patente (7) se indica que se logran altas calidades de reparación al proyectar partículas con una distribución de tamaños estrecha. Por un lado, al eliminar las fracciones más gruesas de las partículas refractarias se facilita el flujo del polvo de soldadura a través del sistema de transporte, se obtiene mejor calidad superficial y disminuye el rebote y, por otro, eliminando los finos aumenta la eficiencia de las reacciones de oxidación debido a que los agregados refractarios finos pueden generar una acción de barrera recubriendo las partículas oxidables e impidiendo que el oxígeno llegue a su superficie.

Los primeros revestimientos refractarios reparados por soldadura cerámica fueron los construidos con ladrillos de sílice, específicamente para la industria del vidrio. La composición del polvo de soldadura para la reparación de estos revestimientos consiste de sílice $\left(\mathrm{SiO}_{2}\right)$ en sus formas polimórficas cristalinas, cristobalita y tridimita, o en estado vítreo y el combustible sólido agregado son partículas muy finas de silicio $(\mathrm{Si})$ y/ o aluminio (Al) en pequeñas cantidades que reaccionan con el oxígeno según las ecuaciones [1] y [2] (2).

$$
\begin{aligned}
& \mathrm{Si}+\mathrm{O}_{2}{ }^{\circledR} \mathrm{SiO}_{2}+210 \mathrm{kcal} / \mathrm{mol} \\
& 2 \mathrm{Al}+3 / 2 \mathrm{O}_{2}{ }^{\circledR} \mathrm{Al}_{2} \mathrm{O}_{3}+408 \mathrm{kcal} / \mathrm{mol}
\end{aligned}
$$

Mezclas de silicio y aluminio también se emplean como combustible con otros óxidos refractarios como zirconia, magnesia, alúmina, sillimanita y mullita. El uso de aluminio en las mezclas de soldadura cerámica es adecuado cuando existe la necesidad de realizar reparaciones a temperaturas relativamente bajas $\left(600-900^{\circ} \mathrm{C}\right)$ debido a que puede promover la iniciación de la fusión a bajas temperaturas. Sin embargo, su uso en las mezclas para reparación por soldadura cerámica de sustratos de sílice se ve limitado. Por un lado, en la masa de reparación puede prevalecer una cantidad de alúmina formada por la oxidación del aluminio, mayor a la presente en un refractario de sílice $(<1 \%)$, obteniéndose diferentes propiedades físicas entre el sustrato y la masa de reparación y disminuyendo la eficacia del proceso. Este desajuste químico es producto de la habitual pérdida de material, esencialmente partículas refractarias, que ocurre durante la proyección de la mezcla. Por otra parte, puede conducir a elevadas velocidades de reacción generando sobrecalentamiento de la zona adyacente a la de reacción y disminuyendo la viscosidad, lo cual provoca que la capa depositada fluya con relativa facilidad sobre el sustrato. Cuando esto ocurre, la correcta aplicación de la carga se dificulta produciendo reparaciones altamente porosas y con un acabado superficial pobre (7). Una composición típica de una mezcla de soldadura cerámica para la reparación de revestimientos refractarios de sílice es: $87 \%$ $\mathrm{SiO}_{2}, 12 \% \mathrm{Si}, 1 \% \mathrm{Al}$.

A través del diseño de distintos polvos de soldadura se pueden obtener masas de reparación con diferentes propiedades. $\mathrm{El}$ agregado de distintos materiales $(\mathrm{CaO}, \mathrm{MgO}$, $\mathrm{FeO})$ puede mejorar ciertas propiedades de las reparaciones por incorporación de los óxidos formados por oxidación del combustible sólido en una red cristalina diferente a la del material base. Por ejemplo, para la reparación de refractarios de sílice, el agregado de magnesia (5-8\%) en la mezcla de soldadura permite la formación de una masa de reparación en la cual al menos un porcentaje de la sílice formada por la oxidación del silicio se incorpora en una red cristalina del tipo fosterita, mejorando de esta manera la refractariedad de la reparación. Si además de magnesia, la mezcla contiene aluminio, la sílice formada se puede incorporar en una red tipo fosterita y/o tipo espinela y/o tipo cordierita. En este último caso se mejora la resistencia al choque térmico (12). Además de una mejora en las propiedades, se ha encontrado que el agregado de estos aditivos mejora la adhesión de la reparación al sustrato durante la operación del horno cuando la temperatura de reparación es baja $\left(\sim 700^{\circ} \mathrm{C}\right)$ o en el caso de refractarios de sílice de alta densidad (porosidad $=14-18 \%$ ) usados en ciertos hornos de coque. Por ejemplo, se ha encontrado que con el agregado de 7,4\% de magnesia a la mezcla de soldadura la masa de reparación obtenida $\left(750^{\circ} \mathrm{C}\right)$ se adhiere eficientemente al refractario de sílice de alta densidad (12).

Otros tipos de mezclas de soldadura se aplican, aunque en menor grado, en otros campos de la industria: materiales basados en magnesia $(\mathrm{MgO})$ y / o alúmina $\left(\mathrm{Al}_{2} \mathrm{O}_{3}\right)$ y materiales de carburo de silicio $(\mathrm{SiC})$ se emplean en la reparación de 
revestimientos refractarios de cucharas en las industrias del acero y del cobre y en la reparación de refractarios en la industria de los metales no-ferrosos, respectivamente (4).

\subsection{Evaluación de las reparaciones}

Para evaluar las masas de soldadura habitualmente se emplean técnicas en las que se miden su resistencia a la compresión en frío, permeabilidad de gases, densidad y porosidad aparentes, porcentaje de partículas oxidables sin reaccionar (4), pero no existen métodos normalizados para medir la adhesión en la interfase sustrato/masa de soldadura debido a las severas complicaciones experimentales, más aún, para su evaluación en caliente. Resulta así habitual realizar una caracterización microestructural de la interfase sustrato/ masa de soldadura con vistas a evaluar cualitativamente y con fines comparativos la adhesión de la masa de soldadura al sustrato refractario. En la Tabla 1 se presentan algunas propiedades de masas de soldadura obtenidas utilizando mezclas de soldadura cerámica para reparar refractarios de sílice (4). A partir de estos datos, se desprende que se pueden obtener masas de reparación con propiedades específicas que las hacen adecuadas para aplicaciones y exigencias diversas (buenas resistencias al choque térmico o a la corrosión, estabilidad química a distintas temperaturas). Así, en las zonas de los revestimientos refractarios sometidas a choque térmico, como es el caso de las puertas de hornos, es conveniente que la mezcla de soldadura permanezca como $\mathrm{SiO}_{2}$ vítrea luego de la proyección para obtener bajos módulo de Young ( $\mathrm{E} \sim 10-14 \mathrm{GPa})$ y coeficiente de expansión térmica $\left(\alpha<0,1 \%\right.$ a $\left.1000^{\circ} \mathrm{C}\right)$. En este caso, se debe prever que la zona reparada permanezca a baja temperatura $\left(20\right.$ a $\left.1000^{\circ} \mathrm{C}\right)$ para evitar la devitrificación de la reparación obtenida. Las masas de soldadura cristalizadas son aplicables en un rango mayor de temperatura $\left(500\right.$ a $1500^{\circ} \mathrm{C}$ ) y son principalmente usadas en hornos de coquización y hornos de vidrio debido a que poseen baja permeabilidad de aire, lo cual previene la percolación de carbón en la atmósfera reductora del horno de coque o la infiltración de álcalis en los hornos de vidrio. También se pueden obtener masas de soldadura de sílice de alta pureza y baja porosidad abierta, lo cual, por un lado, evita la formación de fase líquida cuando se expone a elevadas temperaturas, y estableció en particular para la reparación de refractarios de sílice de calidad KN empleados en la construcción de paredes de hornos de coque siderúrgico, la misma se podría extender en forma directa para el estudio de la reparación de cualquier refractario oxídico, previa determinación de las condiciones particulares de reparación. Sin embargo, su aplicación para la reparación de sustratos refractarios no-oxídicos no resulta simple, en cuyo caso se debería considerar previamente la atmósfera de reparación y el empleo de técnicas adicionales de evaluación relacionadas con las características de los materiales.

La metodología incluye la evaluación tanto del sustrato refractario como de la masa de soldadura e interfase obtenidas permitiendo, en este último caso, una valoración cualitativa de la adhesión. Para realizar la validación de la metodología se compararon los resultados obtenidos en el laboratorio con aquéllos obtenidos a partir del estudio de un ladrillo de sílice post-mortem reparado en una planta siderúrgica.

Se evaluaron polvos de soldadura cerámica comerciales y diseñados que difieren en la granulometría de las partículas refractarias $\left(\mathrm{SiO}_{2}: \mathrm{D}_{50}=0,3-0,9 \mathrm{~mm} ; \mathrm{W}=\left(\mathrm{D}_{90-} \mathrm{D}_{10}\right) / \mathrm{D}_{50}=1,4-2,1\right)$, en el porcentaje relativo de los polimorfos presentes (tridimita/ cristobalita: 0,6 y 2,0$)$ y en el tipo y contenido de las partículas oxidables (Si: $12-14 \%$ yAl: $<<1-2 \%$ ) empleados en la reparación de ladrillos de sílice de calidad KN. Para ello se realizaron ensayos de soldadura cerámica empleando un dispositivo tipo venturi construido bajo diseño propio, previa determinación de las condiciones experimentales, y se determinaron las características de las masas de soldadura obtenidas y de las interfases ladrillo/masa de soldadura.

Se seleccionó una disposición del sustrato refractario a puerta del horno cerrada con un tubo de material refractario en el cual se posiciona el sustrato perpendicularmente a la dirección de proyección, con la doble ventaja de permitir mantener constante la temperatura del sustrato durante la proyección y confinar el polvo de soldadura proyectado al área de reparación (Figura 1).

Las variables experimentales establecidas para la reparación en laboratorio de sustratos de sílice calidad KN con mezclas de soldadura cerámica de tridimita, cristobalita, silicio y aluminio fueron: corriente gaseosa de $\mathrm{O}_{2}$; presión de entrada de $\mathrm{O}_{2}=4,5 \mathrm{bar}$; distancia de proyección $=8 \mathrm{~cm}$; temperatura

TABLA I. PROPIEDADES DE MASAS DE REPARACIÓN BASE SÍLICE (MEYNCKENS, 1.999 (4))

\begin{tabular}{|c|c|c|c|c|}
\hline Masas de reparación & 1 & 2 & 3 & 4 \\
\hline Fases mayoritarias & Sílice vítrea & Cristobalita-tridimita & Cristobalita-tridimita & Cristobalita \\
\hline Contenido de $\mathrm{SiO}_{2}(\%)$ & 90 & 95 & 96 & 99,6 \\
\hline Porosidad abierta $(\%)$ & $10-20$ & $10-20$ & $<10$ & $<10$ \\
\hline Resistencia al choque térmico (ISO 8841) & $>30$ ciclos & - & - & - \\
\hline Modulo de Young [GPa] & $10-14$ & $17-20$ & $17-20$ & $17-20$ \\
\hline Expansión térmica $\left(\%\right.$ a $\left.1000^{\circ} \mathrm{C}\right)$ & $<0,1$ & $<1,2$ & $<1,2$ & $<1,2$ \\
\hline R.U.L $(\mathrm{t} 0,5)$ & - & $1520^{\circ} \mathrm{C}$ & $1550^{\circ} \mathrm{C}$ & $1650^{\circ} \mathrm{C}$ \\
\hline
\end{tabular}

por otro, mejora la resistencia a la corrosión, respectivamente.

\section{METODOLOGÍA DE ENSAYO A ESCALA LABORATORIO}

A partir de estudios realizados en trabajos previos (12-14) se implementó una metodología a escala laboratorio (11) que permite estudiar el proceso de soldadura cerámica en sí mismo y evaluar mezclas de soldadura para su selección con objeto de compra o nuevas mezclas con fines de diseño para aplicaciones específicas. Si bien esta metodología se del sustrato $=1000^{\circ} \mathrm{C}$ (con ciclo de calentamiento programado y enfriamiento libre). En la Figura 2 se muestran fotografías tomadas durante los ensayos de soldadura cerámica realizados en el laboratorio.

Al estudiar los distintos polvos de soldadura se determinó que empleando polvos con granulometría fina y distribución granulométrica amplia se logran reparaciones de calidad superior y porosidad ligeramente menor. Sin embargo, cuando la granulometría de los agregados refractarios que componen la mezcla de soldadura resulta excesivamente fina $\left(D_{50}\right.$ de la 

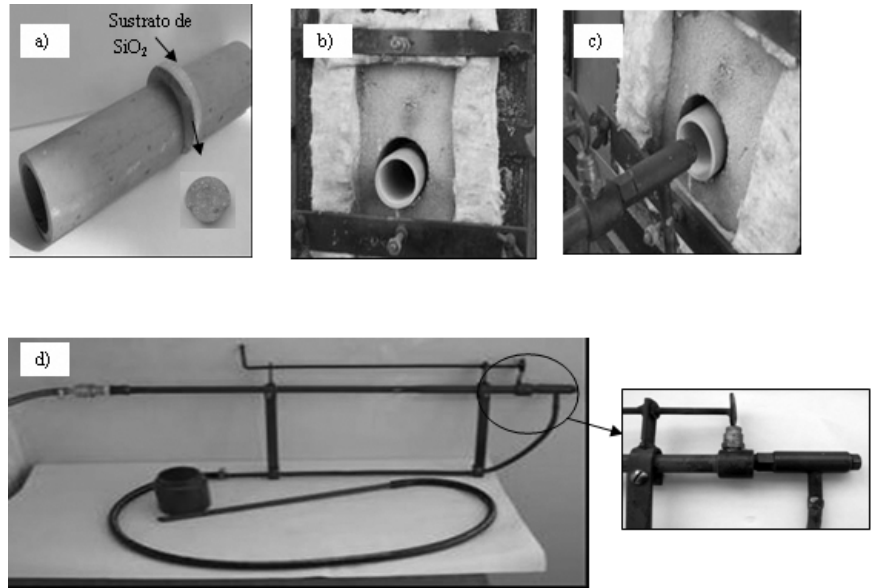

Fig. 1- Disposición de ensayo de soldadura cerámica en laboratorio: detalle con el sustrato (a); tubo con sustrato dentro del horno (b); con el equipo de proyección posicionado para la reparación (c) y dispositivo de proyección diseñado $(\mathrm{d})$.
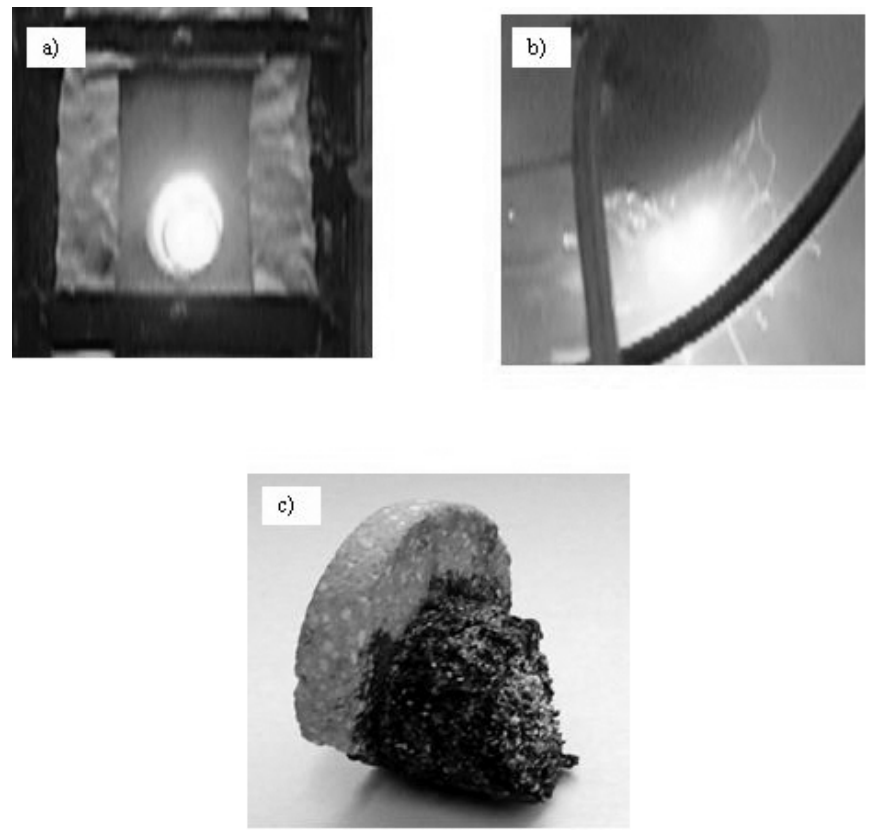

Fig. 2- Ensayo a escala laboratorio: fotografías tomadas antes (a) y durante (b) la proyección de la mezcla de soldadura. Imagen de una reparación obtenida en el laboratorio (c)

distribución total $<0,3 \mathrm{~mm}$ ) se produce, en las condiciones de ensayo empleadas, rebote de la totalidad de las partículas resultando el sustrato refractario dañado por erosión. Como resultado de la minimización del contenido de aluminio de la mezcla de soldadura la operación resulta más controlada sin perjuicio de la reparación obtenida, hecho que concuerda con datos reportados (7).

Las masas de soldadura obtenidas inmediatamente después de las proyecciones resultaron consolidadas y adheridas al sustrato, muy poco cristalinas con alta cantidad de fase vítrea silícea (mayor al incrementar el contenido de silicio en la mezcla) y silicio residual, no pudiéndose descartar contenidos muy bajos de aluminio. Todas las interfases de reparación entre los sustratos y las masas de soldadura presentaron buena continuidad estructural, infiriéndose que en las condiciones de ensayo determinadas a escala laboratorio se ha generado soldadura cerámica: formación de unión cerámica entre la masa de soldadura y el sustrato de sílice. Independientemente de las características de los polvos de soldadura evaluados, los valores porcentuales de las porosidades de estas masas resultaron similares (18-23\%), asociados a poros grandes y/o a poros pequeños del tamaño del orden del de los granos, y caen en el rango de valores reportados para mezclas de soldadura cerámica de base sílice empleadas en la reparación de revestimientos refractarios de hornos de coque (4). Por tratamiento térmico a $1200^{\circ} \mathrm{C}$ (temperatura de operación de los hornos de coque siderúrgico) durante tiempos variables, las masas de soldadura evolucionan hacia las fases cristalinas termodinámicamente estables presentando, ya a las 2,5h de permanencia a la temperatura indicada, un aspecto similar al ladrillo post-mortem que operó en servicio: coloración más clara y estratificación, con las mismas fases cristalinas: cristobalita, tridimita y escasa cantidad de cuarzo junto a silicio residual (DRX). La porosidad de estas masas resultó del orden de la determinada en el ladrillo post-mortem y similar a la de las masas de soldadura sin tratamiento térmico posterior a las proyecciones. Las microestructuras de las masas tratadas térmicamente presentaron granos con bordes más definidos, partículas esféricas de silicio residual de menor tamaño (3) y aumento de la cantidad de puentes sólidos por soldadura cerámica, resultando muy difícil distinguir la interfase de reparación luego del tratamiento térmico. A modo de ejemplo, se presentan en la Figura 3 imágenes obtenidas por SEM de una masa soldadura y de la interfase masa de soldadura/ ladrillo de sílice, esta última indicada con flechas.
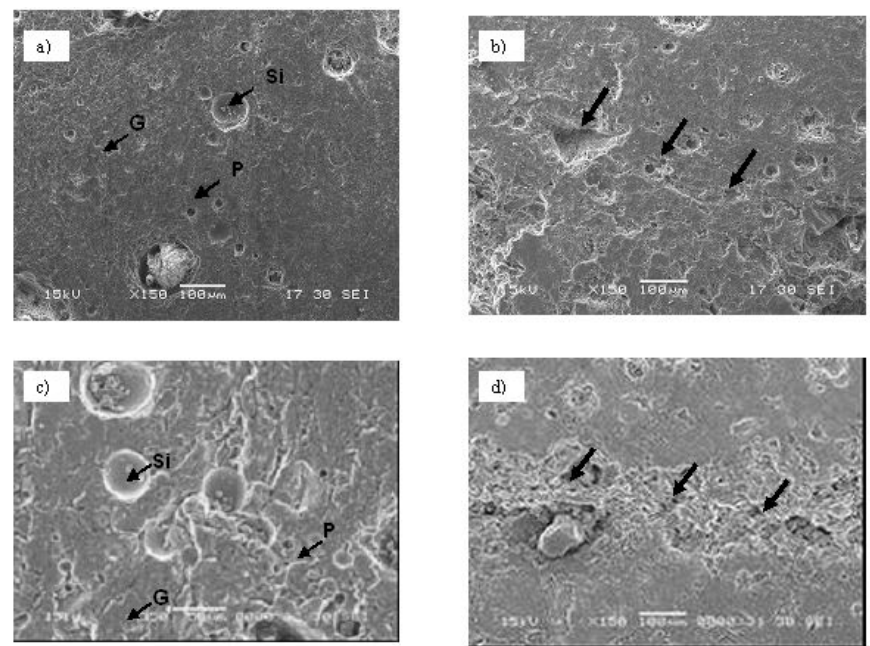

Fig. 3- Imágenes obtenidas por SEM de: masa de soldadura (P: poro, G: grano, Si: silicio) (a) e interfase ladrillo de sílice (zona inferior)/masa de soldadura (zona superior) (b) obtenidas en el laboratorio y de la masa de soldadura (P: poro, G: grano, Si: silicio) (c) e interfase ladrillo de sílice post-mortem (zona inferior) / masa de soldadura post-mortem (zona superior) $(\mathrm{d})$.

\section{CONSIDERACIONES FINALES}

Se han expuesto los aspectos más relevantes del método de soldadura cerámica para la reparación de revestimientos refractarios, tecnología de elevada potencialidad y de actual aplicación en el campo industrial. Así, resulta de sumo interés contar con una metodología a escala laboratorio para ensayos de soldadura cerámica que permita contribuir al conocimiento 
sobre el tema, dar lineamientos para la selección de mezclas con objeto de compra o para la formulación de nuevas mezclas y brindar posibles soluciones a requerimientos concretos del sector industrial.

\section{BIBLIOGRAFÍA}

1. A. R. Hamacher, Durable renovation of defective coke oven walls using the ceramic welding process, World Ceramics, 19-24 (1985).

2. Fosbel, Principios técnicos: Soldadura Cerámica: Un sistema adelantado de arreglo en caliente, (1986).

3. B, Titterington, Coke oven repairs within british steel using silica welding, I \& SM, 5, 67-78 (1992).

4. J. P. Meynckens, Ceramic Welding: Techniques and improvements for glass furnaces, Unified International Technical Conference on Refractories, Berlín, Alemania, 238-240 (1999).

5. C. Zvosec, J. Briggs, Ceramic welding: A new approach to the production of refractory repair masses, I \& SM, 9, 43-46 (1988).
6. K. Pendleton, Ceramic Welding: Performance by Design, Ceramic Industry, 7, 29-31 (2001).

7. L.E.J. Tueker, K. Dunderdale, D.K. Hurran, R.G. Everitt, Ceramic welding US Patent 5,380,563 (10-01-1995).

8. B. Dueker, J. Smart, Ceramic welding (Oxythermie) - the refractory life support system, Cokemaking International, 2, 53-56 (1990).

9. A. Zivkovic, Ceramic welding method with monitored working distance, US Patent 5,378,493 (03-01-1995).

10. J. P. Meynckens, B. Somerausen, A process of preparing a crystalline siliceous refractory mass, US Patent 5,780114 (01-05-2004).

11. H. E. Lobato, Soldadura cerámica para la reparación de hornos de coque siderúrgico, Proyecto Final de la carrera Ingeniería en Materiales. Facultad de ingeniería, Universidad Nacional de Mar del Plata (2006).

12. J. P. Meynckens, L.P. Mottet, Process and mixture for forming a coherent refractory mass on a surface, US patent 5,866,049 (02-02-1999).

13. H. Lobato, M. A. Camerucci, A. L. Cavalieri, Soldadura cerámica para la reparación de refractarios de hornos industriales, Anales $50^{\circ}$ Congreso Brasilero de Cerámica, Blumenau, Brasil, 8-12, 1-13 (2006).

14. H. Lobato, M. A. Camerucci, A. L. Cavalieri, Diseño y evaluación de mezclas de soldadura cerámica, Actas CONAMET-SAM 2006, Santiago, Chile (2006).

Recibido: 19.03.07

Aceptado: 27.07.07 